\title{
Manejo de Tumores de Mediastino, Serie de Casos.
}

\section{Manangement of Mediatinum Tumors, Case series.}

\section{Carlos Marengo Baquerizo 1* iD, Mauricio Lara Perlaza1, Pamela Macías Fernández1.}

*Correspondencia: cmarengo@hotmail.es

Teléfono [593] 043718700

Conflicto de intereses: Los autores declaran no tener conflictos de intereses.

Fondos: Ver la página 6

Recibido: 3 Febrero 2017 Aceptado: 20 Junio 2017 Publicado: 30 Abril 2018

Membrete bibliográfico: Marengo C, Lara M, Macías P. Manejo de Tumores del Mediastino, serie de casos. Rev. Oncol. Ecu 2017;28(1):1-8.

DOI: https://doi.org/10.33821/242

Copyright Marengo, et al. Este artículo es distribuido bajo los términos de Creative Commons Attribution License, el cual permite el uso y redistribución citando la fuente y al autor original.
1. Instituto Oncológico Nacional "Dr. Juan Tanca Marengo" Solca, GuayaquilEcuador, servicio de Ciurgía Oncológica.

\section{Resumen}

Introducción: A pesar del difícil acceso anatómico para los tumores de mediastino, la resección quirúrgica sigue siendo el mejor enfoque diagnóstico y terapéutico. El objetivo de la presente serie de casos presentamos la experiencia de un centro oncológico en el abordaje de tumores del mediastino y sus resultados.

Métodos: En el departamento de Jefatura de Cirugía Oncológica del Instituto Oncológico nacional de Solca-Guayaquil, durante los meses de Enero del 2013 a Enero 2017 se realizó un estudio descriptivo, retrospectivo. Se analizaron todos los casos de pacientes derivados del área de pre admisión con diagnóstico inicial de tumor de mediastino, a los cuales previo a realizarles marcadores tumorales, Tomografía de Tórax, y a quienes se les realizó como método diagnóstico y en algunos casos terapéutico con abordaje quirúrgico. Se excluyeron pacientes con neoplasias de origen secundario, con historias clínicas incompletas que imposibilitaron el análisis. Se estudiaron las variables sexo, edad, Tipo de Técnica quirúrgica, localización del tumor, diagnostico histopatológico y mortalidad perioperatoria. El análisis estadístico realizado fue descriptivo.

Resultados: Se evaluaron 22 pacientes con diagnóstico tumor de mediastino, con una edad media de 60 años. Fueron 13 hombres (59\%). Catorce pacientes con lesión maligna, 8 fueron operados con resección tumoral y 6 se sometieron a biopsia. Se reportaron fallecimientos post-operatorios hasta los 30 días postquirúrgicos en 5 casos (22.7\%). Las intervenciones quirúrgicas realizadas con más frecuencia fueron toracotomías derechas con toma de biopsia o resección tumoral 7 casos (31.8 \%). En relación al resultado anatomopatológico de los tumores de mediastino reportados más de la mitad constituyeron Adenocarcinomas 14 casos (63.6\%).

Conclusión: Los pacientes portadores de neoplasia benigna en esta serie tuvieron una evolución posquirúrgica satisfactoria. El adenocarcinoma fue el tumor maligno más frecuente en esta serie.

Palabras Claves: MEDIASTINO, NEOPLASIAS, ADENOCARCINOMA.

DOI: $10.33821 / 242$ 


\section{Abstract}

Introduction: Despite the difficult anatomical access for mediastinal tumors, surgical resection remains the best diagnostic and therapeutic approach. The aim of this case series is to present the experience of a cancer center in the treatment of mediastinal tumors and their results.

Methods: In the Department of Oncology Surgery Headquarters of the National Oncology Institute of Solca-Guayaquil, during the months of January 2013 to January 2017 a descriptive, retrospective study was carried out. We analyzed all cases of patients derived from the area of pre-admission with initial diagnosis of mediastinal tumor, which prior to performing tumor markers, Thorax Tomography, and who were performed as a diagnostic method and in some cases therapeutic with surgical approach. We excluded patients with neoplasms of secondary origin, with incomplete medical records that made the analysis impossible. The variables sex, age, type of surgical technique, tumor location, histopathological diagnosis and perioperative mortality were studied. The statistical analysis performed was descriptive.

Results: Twenty-two patients with a mediastinal tumor diagnosis were evaluated, with a mean age of 60 years. There were 13 men (59\%). Fourteen patients with malignant lesions, 8 were operated with tumor resection and 6 underwent biopsy. Post-operative deaths were reported up to 30 days after surgery in 5 cases $(22.7 \%)$. The most frequent surgical interventions were right thoracotomies with biopsy or tumor resection 7 cases $(31.8 \%)$. In relation to the anatomopathological result of mediastinal tumors reported more than half Adenocarcinomas were 14 cases (63.6\%).

Conclusion: The patients with benign neoplasm in this series had a satisfactory postoperative evolution. Adenocarcinoma was the most frequent malignant tumor in this series.

Keywords: MEDIASTINE, NEOPLASMS, ADENOCARCINOMA.

DOI: $10.33821 / 242$

\section{Introducción}

A pesar del difícil acceso anatómico para los tumores de mediastino, la resección quirúrgica sigue siendo el mejor enfoque diagnóstico y terapéutico. La aceptación generalizada de la toracoscopía asistida por video (VATS) está restringida por la naturaleza limitante de los instrumentos y la visualización subóptima, por lo que la cirugía abierta continúa siendo la estandarización para este fin.

Debido a que la ubicación de los tumores mediastinales está limitada por los espacios anatómicos naturales del mediastino, los procedimientos se clasifican en toracotomía anterior, posterior y media, cada una de ellas con sus peculiaridades quirúrgicas específicas y grado de dificultad diferente. Incluso cada espacio tiene zonas estrechas y remotas por lo que recientemente se han reportado cirugías mínimamente invasiva asistidas por robot para acceso a estas regiones.

La frecuencia de tumores es más prevalente en el mediastino anterior >del $50 \%$ de los casos ocupan este espacio, estos son Timoma, Linfomas, tumores de células germinales y de tiroides. Sin embargo debido a que el mediastino es un compartimiento torácico que alberga múltiples estructuras anatómicas, condiciona una amplia gama de tumores 
malignos y benignos, quísticos y sólidos, primarios y secundarios. Así mismo, estos exhiben muchas variedades histológicas en relación a la diversidad de órganos y tejidos que les dan origen [1, 2]. En esta serie de casos presentamos la experiencia de un centro oncológico en el abordaje de tumores del mediastino y sus resultados.

\section{Materiales y Métodos}

En el departamento de Jefatura de Cirugía Oncológica del Instituto Oncológico nacional de Solca- Guayaquil, durante los meses de Enero del 2013 a Enero 2017 se realizó un estudio descriptivo, retrospectivo.

Se analizaron todos los casos de pacientes derivados del área de pre admisión con diagnóstico inicial de tumor de mediastino, a los cuales previo a realizarles marcadores tumorales, Tomografía de Tórax, y a quienes se les realizó como método diagnóstico y en algunos casos terapéutico con abordaje quirúrgico.

Los criterios de inclusión fueron pacientes de ambos sexos mayores de 18 años, con diagnóstico presuntivo inicial de tumor mediastinal y que recibieron tratamiento quirúrgico en el departamento de Jefatura de Cirugía Oncológica de Solca-Guayaquil. Se excluyeron pacientes con neoplasias de origen secundario, con historias clínicas incompletas que imposibilitaron el análisis.

Se estudiaron las variables sexo, edad, Tipo de Técnica quirúrgica, localización del tumor, diagnostico histopatológico y mortalidad perioperatoria. El análisis estadístico realizado fue descriptivo.

\section{Resultados}

Se evaluaron 22 pacientes con diagnóstico tumor de mediastino, con una edad media de 60 años, en el rango de 20 a 79 años. Fueron 9 mujeres $40.9 \%$ y 13 hombres (59\%). Se presentaron 2 casos ( $9 \%$ ) en menores de 30 años; 8 casos ( $36.3 \%$ ) entre 30 a 59 años; y 12 casos $(54.5 \%)$ en $>60$ años. Los pacientes portadores de neoplasia benigna en esta serie tuvieron una evolución posquirúrgica satisfactoria.

Catorce pacientes con lesión maligna, 8 fueron operados con resección tumoral y 6 se sometieron a biopsia, se los remitió a oncología clínica para manejo coadyuvante. Se reportaron fallecimientos post-operatorios hasta los 30 días postquirúrgicos en 5 casos (22.7\%).

Las intervenciones quirúrgicas realizadas con más frecuencia fueron toracotomías derechas con toma de biopsia o resección tumoral (Tabla 1). En relación al resultado anatomopatológico de los tumores de mediastino reportados más de la mitad constituyeron Adenocarcinomas (Tabla 2). 
Tabla 1. Intervenciones de abordaje quirúrgico de tumores del mediastino

\begin{tabular}{|l|c|}
\hline Intervenciones quirúrgicas & $\mathbf{N}=22(100 \%)$ \\
\hline Toracotomía derecha+ biopsia & $7(31.8 \%)$ \\
\hline Toracotomía derecha+ resección tumoral & $5(22.7 \%)$ \\
\hline Toracotomía diagnóstica & $4(18.1 \%)$ \\
\hline Toracotomía izquierda+ resección tumoral & $3(13.6 \%)$ \\
\hline Toracotomía izquierda + biopsia & $3(13.6 \%)$ \\
\hline
\end{tabular}

Tabla 2. Reporte histopatológico de etiología tumoral mediastinal.

\begin{tabular}{|l|c|}
\hline Intervenciones quirúrgicas & $\mathbf{N}=22(100 \%)$ \\
\hline Adenocarcinoma & $14(63.6 \%)$ \\
\hline Granuloma tuberculoso & $2(9 \%)$ \\
\hline Fibrosis benigna & $1(4.5 \%)$ \\
\hline Mieloma & $1(4.5 \%)$ \\
\hline Quiste mediastinal & $1(4.5 \%)$ \\
\hline Linfoma no Hodking & $1(4.5 \%)$ \\
\hline Tumor Neuroendócrino & $1(4.5 \%)$ \\
\hline Schawnoma Benigno & $1(4.5 \%)$ \\
\hline
\end{tabular}

\section{Discusión}

En la presente serie de casos, los pacientes con tumor de mediastino fueron la mayoría de sexo masculino, en relación con los artículos publicados por la comunidad científica [3-6]. En cuanto a la edad, aunque se reporta que se presenta en cualquier etapa de la vida, en este reporte en los 5 años del registro, la edad media de estos pacientes es de 60 años.

Las estrategias terapéuticas quirúrgicas de los tumores mediastínicos son tan variados como la diversidad patológica; dependiendo de la experiencia del cirujano y de su equipo; localización anatómica, etiología de la enfermedad, recursos con los que se contó y comorbilidades que presentaron.

Las técnicas más comunes para obtener la histología de los tumores de mediastino incluyen biopsia por aspiración con aguja fina guiada por imagen, la biopsia quirúrgica a través de varios enfoques, y la resección completa; en varios estudios [7-12] se prefiere la biopsia por aspiración con aguja fina guiada por imagen, pero dada la posibilidad de obtener muestras insuficientes a $1 \mathrm{~mm}$ con las cuales no se puede precisar el diagnóstico histológico, se termina recurriendo a otros procedimientos como la mediastinostomía anterior, mediastinoscopía, toracoscopía, lo cual concuerda con lo que se realiza en este reporte, en el cual el abordaje quirúrgico más frecuente para la toma de la biopsia fue la mediastinostomía anterior. 
La incisión quirúrgica predominante en esta serie de casos es la toracotomía exploradora antero lateral, con sus grandes ventajas y exposición, que permitió una toma adecuada de muestra de tejido y a un diagnóstico adecuado. En ningún caso se recurrió a estereotomía media.

En este reporte de 22 casos el resultado patológico predominante en los tumores mediastinales fue el Adenocarcinoma, que difiere de la literatura internacional, de los cuales los más frecuentes son los timomas, teratomas y linfomas.

Se reportaron cinco fallecidos en esta serie, por lo que es importante en las neoplasias de mediastino un diagnóstico temprano que permita seleccionar la estrategia terapéutica más adecuada de los pacientes afectados, sobre todo con neoplasias malignas.

Futuras investigaciones deberán abordar seguimientos, supervivencias, técnicas con un grupo control para el mejor estudio del tema.

\section{Conclusiones}

En conclusión el manejo quirúrgico de los tumores mediastinales continúa siendo un reto para el paciente que lo padece y el equipo quirúrgico, debido a la diversidad de la patología y la complejidad del tratamiento. Los pacientes portadores de neoplasia benigna en esta serie tuvieron una evolución posquirúrgica satisfactoria. El adenocarcinoma fue el tumor maligno más frecuente en esta serie.

Podemos finalmente establecer que de forma general, se obtuvieron resultados que concuerdan, en gran medida, con otros estudios realizados sobre manejo quirúrgico de tumores mediastinales, y el abordaje quirúrgico para el diagnóstico, concuerda con la mayoría de las publicaciones de la comunidad científica, donde en mayor frecuencia se utiliza la mediastinostomía anterior.

\section{Agradecimientos}

Se reconoce a las personas que participaron indirectamente en el estudio tales como el personal técnico y otras en general del Instituto Oncológico Nacional "Dr. Juan Tanca Marengo", Solca- Guayaquil. 


\section{Información adicional}

Nota del Editor

La Revista Oncología Ecu permanece neutral con respecto a los reclamos jurisdiccionales en mapas publicados y afiliaciones institucionales.
Abreviaturas

Solca: Sociedad de Lucha Contra el Cáncer.

Archivos Adicionales

Ninguno declarado por los autores.

\section{Fondos}

Los fondos de la investigación fueron propios de los autores del presente artículo.

\section{Disponibilidad de datos y materiales}

Existe la disponibilidad de datos bajo solicitud al autor de correspondencia. No se reportan otros materiales.

\section{Contribuciones de los autores}

$\mathrm{MV}, \mathrm{NL}$, realizaron la idea de investigación, revisión bibliográfica, el análisis crítico del artículo. $\mathrm{RQ}$ realizó la recolección de datos, análisis estadístico, escritura del artículo. MV realizó las correcciones. Todos los autores leyeron y aprobaron la versión final del artículo.

\section{Aprobación de ética y consentimiento para participar}

No aplica ya que es un estudio de bases de datos.

\section{Consentimiento para publicación}

No aplica.

\section{Información de los autores}

Carlos Marengo Baquerizo, Médico cirujano oncólogo, Jefe de Servicio de Cirugía Oncológica del Instituto Oncológico Nacional "Dr. Juan Tanca Marengo".-SolcaGuayaquil. (iD) https://orcid.org/0000-0002-0749-5080 
Mauricio Lara Perlaza, Médico Cirujano Oncólogo, tratante del Servicio de Cirugía Oncológica del Instituto Oncológico Nacional "Dr. Juan Tanca Marengo".Solca- Guayaquil.

Pamela Macías Fernández, cirujano general del Servicio de Cirugía Oncológica del Instituto Oncológico Nacional "Dr. Juan Tanca Marengo". Solca- Guayaquil

\section{Revisiones por pares}

Acceda a la revisión de pares académicos en el siguiente enlace: https://publons.com/review/3791971

\section{Referencias}

Abreviaturas en la referencias DOI: Digital Object Identifier

PMID: PubMed Identifier

SU: Short URL
1. Guzmán F, Morales D, Guerrero Y. Evaluación, Diagnóstico y tratamiento quirúrgico de Neoplasias de Mediastino. Revista Venezolana de Oncología 2006:18(1):SN1. SU: goo.gl/hfWvQa

2. Rodriguez C, Arce C, Amarilla L, Andreo T, Araujo D, Arzamendia L, et al. Características clínicas y Patológicas de los Tumores de Mediastino de un Hospital Universitario. Rev. Cir. Parag. 2013;37(2):2225. SU: goo.gl/nbiVHd

3. Perez A, De Armas F, Sabatier C, Estupiñán B. Tumores de Mediastino estudio de 5 años y 3 meses en el Hospital CIMEQ. Actas Hispanoamericanas de Patología 2006;1-4. SU: goo.gl/S1yBec

4. Salazar S, Calderon F, Maya E, Poveda S. Tumores Mediastinicos Gigantes, estudio de una década, Hospital Carlos Andrade Marín, Quito. Revista Ciezt. Clinica \& Cirugia 2012;12(1):1-7. SU: goo.gl/FD2ukZ

5. Li Y, Wang J. Experience of videoassisted thoracoscopic resection for posterior mediastinal nerogenic tumours a retrospective analysis of 58 patients. ANZ J Surg. 2013 Sep;83(9):664-8. DOI: 10.1111/j.1445-2197.2012.06174.x

6. Navarro G, Barba J, Benitez C, Bravo L, Gavidia J. Abordaje Videotoracoscopico de Tumores Mediastinicos. Analisis de 16 casos. Rev Oncol Ecu 2015;25(1).

7. Santillan-Doherty P. Tumores Mediastinales. Revista de Investigación clínica 2006;58(3):245-253. SU: goo.gl/E83cyY

8. Torres T, Herrera D, Galvez M, Moran E. Masas Mediastinales Epidemiologia y decisiones estratégicas. Experiencia 13 años. Neumol Cir Torax 2016;75(4):268-274. SU: goo.gl/oG1mK8

9. Berry MF, Jeff JR, Muller NL. Evaluation of mediastinal masses. Literature review current through. Sept. 2015 Disponible en:https://www.uptodate.com/.../approach-to-the-adult-patiente-with-a mediastinal-Masses

10. Rodriguez A, Capin N, Torres L. Tumores de Mediastino: Informe sobre 29 pacientes. Revista Cubana de Cirugía 2008;47(4):1-7. SU: goo.gl/S5uo2W

11. Whitson B, Groth S, Duval S, Swanson S, Maddaus M. Surgery for early-stoge non-small cell lung cancer: a systematic review of the video-assisted thoracoscopic surgery versus thoracotomy approaches to lobectomy. Ann Thorac. Surg. 2008;86(6):2008-16. DOI: 10.1016/j.athoracsur.2008.07.009

12. Fernandez- Arrieta A, Gutierrez E, Jiménez-Quintana N, Klele-Espinosa C, Ramos-Clason EC. Epidemiología de los tumores mediastinales en el hospital universitario del caribe. Tesis de especialidad en cirugía. Universidad de Cartagena. SU: goo.gl/k2Jyk9 
13. Pastrana T, De lima L, Pons JJ, Centeno C. Atlas de Cuidados Paliativos en Latinoamérica. Asociación Latinoamericana en Cuidados Paliativos. SU: goo.gl/j99URx 